medical education meetings with BMA representation, leading to reformation through widespread changes providing better support and universal access to services.

\section{COLLABORATION BETWEEN HOSPITAL LEADERHSIP AND FRONTLINE CLINICAL STAFF: IMPORTANT IN A SAFETY CULTURE}

${ }^{1}$ Eseme Ebai ${ }^{*},{ }^{2}$ Daniel Shu, ${ }^{3}$ Isabelle Feldhaus, ${ }^{4}$ Girish Motwani, ${ }^{7}$ Guifo Marc Leroy, ${ }^{5}$ Ebai Myriam, ${ }^{6}$ Catherine Juillard. 'Division of Plastic, Reconstructive and Aesthetic Surgery, Geneva University Hospitals, Switzerland; 'Lead Higher Institute, Bethesda Hospital, Yaoundé, Cameroon; ${ }^{3}$ Department of Global Health and Population, Harvard T.H. Chan School of Public Health, Boston, Massachusetts, USA; ${ }^{4}$ Center for Global Surgical Studies, Department of Surgery, University of California, San Francisco, San Francisco, California, USA; ${ }^{5}$ Elites Gifted Hands Medical Center, Yaoundé, Cameroon; ${ }^{6}$ Department of Surgery, University of California, Los Angeles, California, USA; ' University Hospital Center of Yaounde, Cameroon

\subsection{6/leader-2021-FMLM.12}

Background The rate of harm resulting from unsafe care is known to be high in African health systems and there is a significant need to improve patient safety culture through the active involvement of healthcare leaders in creating an environment that enhances organizational culture around patient safety in low and middle income settings.

Methods A questionnaire developed from the Agency for Healthcare Research and Quality (AHRQ) was used to assess the organizational culture of patient safety among 120 frontline clinical staff for four health care facilities in Yaounde, Cameroon. Ethical clearance was obtained for the study.

Results Most participants (85\%) responded that they clearly know the mission, vision, and goals of their institution. 97 $(80.7 \%)$ of respondents agreed or strongly agreed that they receive strong emphasis from their hospital hierarchy for safe care. Participant responses varied by facility on the perceived culture of collaboration between management and frontline staff $(\mathrm{p}=0.0374)$. Overall, only $43.8 \%$ of respondents felt that there was strong collaboration between management and medical staff. Overall, $47.5 \%$ of all respondents expressed that they would not feel safe being treated in their own hospital. The majority $(76.7 \%)$ have no notion of patient safety and $94.2 \%$ expressed the desire for more training on the topic.

Although there is strong emphasis from leadership for safe care, the low awareness on the notion of patient safety, low collaboration between the leadership and frontline staff and the fact most frontline clinical staffs will not feel safe being treated in their own hospitals, are important indicators that reveal an unhealthy patient safety culture in the hospital.

Conclusion Hospital Leadership has a major role to play in enhancing a patient safety organizational culture. Collaboration between the leadership and frontline staff is indispensable for a successful outcome. These setbacks can be reduced with appropriate training.

\section{CREATION OF A FREE, ACCESSIBLE EVENT FIGHTING FAKE NEWS REGARDING COVID-19}

'Daniella Nunes Pereira, ${ }^{2}$ Nathalia Sernizon Guimarães, ${ }^{3}$ Thaís Lorenna Souza Sales, ${ }^{4}$ Cristiane dos Santos Dias, ${ }^{5}$ Virgínia Mara Reis Gomes, ${ }^{6}$ Milena Soriano Marcolino. 'Undergraduate Medical Student. Universidade Federal de Minas Gerais. Avenida Professor Alfredo Balena, 190, Belo Horizonte, Brazil; ${ }^{2}$ Post-Doctoral Resident at Postgraduate Program in Health Science: Infectious Diseases and Tropical Medicine, Universidade Federal de Minas Gerais. Avenida Professor Alfredo Balena 190, sala 503, Belo Horizonte, Brazil. Fellowship of Institute for Health Technology Assessment (IATS/CNPq). Rua Ramiro Barcelos, 2359. Prédio 21 | Sala 507, Porto Alegre, Brazil; ${ }^{3}$ PhD student in the Graduate Program in Health Sciences. Universidade Federal de São João Del-Rei. R. Sebastião Gonçalves Coelho, 400, Divinópolis, Brazil; ${ }^{4}$ Pediatrician and Professor. Department of Pediatrics, Universidade Federal de Minas Gerais. Avenida Professor Alfredo Balena 190, sala 503, Belo Horizonte, Brazil; ${ }^{5}$ Undergraduate Medical Student. Centro Universitário de Belo Horizonte (UniBH). Av Professor Werneck, 1685, Belo Horizonte, Brazil; ${ }^{6}$ Associate Professor and Internal Medicine Physician. Department of Internal Medicine, Medical School; and Telehealth Center, University Hospital, Universidade Federal de Minas Gerais. Avenida Professor Alfredo Balena 190, sala 246, Belo Horizonte, Brazil. Researcher. Institute for Health Technology Assessment (IATS/CNPq). Rua Ramiro Barcelos, 2359. Prédio 21 | Sala 507, Porto Alegre, Brazil

\subsection{6/leader-2021-FMLM.13}

Introduction Our aim was to describe the First Brazilian Congress of Clinical Evidence on COVID-19, which took place in Brazil, where the pandemic has hugely impacted the population.

Methods The organizing committee consisted of a multidisciplinary team of 23 volunteers. As official communication tools, WhatsApp, email, shared folders in Google Drive and online meetings were used. We hoped that the event would boost the fight against fake news and help raise awareness on COVID-19 prevention methods. A major concern of the organizing team was to spread factual knowledge about COVID-19. To this end we had simultaneous translation and interpretation in Brazilian Sign Language to ensure accessibility.

Results In total, 23,573 participants registered for the event, and over 30,000 views on the first day, with an overall reach of 85,000 views for the original audio version (up to September 5th, 2021). At the end of the event, a satisfaction survey with participants showed that expectations were exceeded for $97.5 \%$, and $86.7 \%$ reported acquiring new knowledge about COVID-19.

Discussion The conference was an important opportunity for teamwork and for providing reliable information to Brazil's population. Technology allowed us to reach participants from all Brazilian states and even other countries. However, the majority of speakers were physicians and some healthcare areas were not represented. The team decided to host a Second Congress, including speakers from different specialties to account for knowledge gaps. With the increasing levels of contamination and deaths by COVID-19 in Brazil and the lack of effective public policies to combat the virus, the dissemination of good, reliable information concerning COVID-19 was urgent.

Conclusion Hosting a free, online, evidence-based event to disseminate knowledge on COVID-19 is feasible, and it is possible to engage a large number of participants. 